\title{
Super-Recognizers: More Consistent or Qualitatively Different Psychophysical Profiles?
}

\author{
Jeffrey D Nador ${ }^{1}$, Matteo Zoia ${ }^{1}$, Matthew V Pachai2 \& Meike Ramon ${ }^{1 *}$ \\ ${ }^{1}$ Applied Face Cognition Lab, University of Fribourg, Fribourg, Switzerland; 2Perceptual Neuroscience Laboratory, York University, \\ Toronto, Canada; Correspondence: meike.ramon@gmail.com
}

Facial identity matching ability varies widely, ranging from severely deficient prosopagnosics (who exhibit profound impairments in face cognition) to so-called Super-Recognizers (SRs), possessing exceptional capacities for processing facial identity. Yet, despite the often consequential nature of face matching decisions-such as identity verification in security critical settings-ability assessments rely on simple performance metrics on a handful of heterogeneously related subprocesses, or in some cases only a single measured subprocess. Unfortunately, methodologies of this ilk leave contributions of stimulus information to observed variations in ability largely unspecified. Moreover, they are inadequate for addressing the qualitative or quantitative nature of differences between SRs' abilities and those of the general population. Here, therefore, we sought to investigate individual differences-among SRs identified using a novel conservative diagnostic framework, and neurotypical controls - by systematically varying retinal availability, bandwidth, and orientation of faces' spatial frequency content over two face matching experiments. Psychophysical evaluations of these parameters' contributions to ability reveal that SRs more consistently exploit the same spatial frequency information, rather than suggesting qualitatively different profiles between normal observers and SRs. These findings stress the importance of optimizing procedures for SR diagnosis to include measures of individuals' consistency.

\section{Introduction}

Ability in matching images of unfamiliar face identities varies widely between neurotypical adults (Fox \& Bindeman, 2020; Estudillo \& Bindemann, 2014; Fysh \& Bindemann, 2017; Stacchi et al, 2020; Fysh et al., 2020). At the upper end of this spectrum are SuperRecognizers (SRs), originally reported by Russel, Duchaine and Nakayama (2009) as possessing exceptional facial identity processing capabilities across a range of subprocesses, including face matching, recognition, and identification. Presently, though, there is limited empirical evidence concerning the factors underlying such extreme abilities (for a recent review see Ramon, Bobak \& White, 2019). To date, two aspects have hindered genuine progress in the scientific endeavor of understanding the mechanisms underlying superior face cognition skill: (i) the use of varied and inappropriate diagnostic criteria for assessing such skills; (ii) absence of studies systematically varying the information conveyed by face stimuli themselves.

Diagnosis of superior face processing - that is, of SRs-generally involves application of simple performance criteria on standardized laboratory-based tests. As mentioned, Russell and colleagues' (2009) seminal study reported a small number of SRs who indeed achieved superior performance on three tests of face cognition measuring perceptual matching, old/ 
new recognition and identification. Yet in the intervening time, departing from this multi-test procedure, more recent studies have adopted criteria based on a single test/process measured: performance surpassing two standard deviations above the mean of "normal" controls (Bobak et al., 2016a; Bobak, Pampoulov \& Bate, 2016; Bobak, Dowsett \& Bate, 2016) on the long version of the Cambridge Face Memory Test (CFMT+) (Russell et al., 2009); or "a previous face recognition test score that placed [them] above a super-recognizer benchmark [which] could have been any in the super-recognizer literature" (Phillips et al., 2018, Supplementary Information, p. 2). Minimally, though, multiple measures should be applied in diagnoses to ensure that any evidence of superior performance within an individual is consistent across tasks/subprocesses. That is, if SRs are considered to display superior face processing abilities, generally, then their assessment should assess the consistency of their performance relative to normal observers across tasks.

The single-criterion approach to SR diagnosis is problematic for at least two reasons. First, it is well-established that superior performance on one face cognition task/subprocess does not generalize to others (Bobak et al., 2016b; Bate et al., 2018; Lander, Bruce \& Bindeman, 2018; Stacchi et al., 2020; Fysh, 2018; Fysh et al., 2020), so criterion performance on any single task/sub-process should not be considered sufficient evidence of processing superiority to warrant generalizing across tasks/sub-processes. Second, some tests that have been used as a criterion for superior skill are either inappropriate, or often insufficiently sensitive for SR diagnosis. This applies to the short (72-item) version of the CFMT, which was developed for the diagnosis of developmental prosopagnosia (Duchaine \& Nakayama, 2005), and extends to the CFPT (Duchaine \& Nakayama, 2006) and GFMT (Burton et al., 2010) two tests for which individuals with developmental prosopagnosia can achieve normal performance (Russel et al., 2009; White et al., 2017).

These diagnostic issues have probably led to false positive SR diagnoses among studies adopting them, in turn contributing to the heterogeneity reported in the SR literature (Ramon et al., 2019). Therefore, reduction of false positives - and by extension the advancement of our understanding of SRs' abilities - requires a more conservative framework for their diagnosis (Ramon, 2021). Moreover, these criteria should be evaluated within the context of cross-task consistency within observers (Ramon et al., 2019; Stacchi et al., 2020; Fysh et al., 2020), in order to determine whether SRs indeed consistently live up to their namesake in distinguishing themselves from the general population.

Diagnostic issues aside, previous empirical studies of SRs generally fall short with respect to two major issues: failure to systematically vary any parameters across the full face stimulus, and consideration of only simple performance measures. Consequently, these studies are left hard-pressed to answer the currently open question of whether normal observers and SRs differ qualitatively, i.e. due to exploitation of distinct types of information (Schyns, 1998; Gosselin \& Schyns, 2001; Goffaux et al., 2003), or quantitatively, e.g. due to differential exploitation of the same local, featural information available to normal observers (Tardif et al., 2019).

In general, observers' performance on tasks employing systematic variations of stimulus parameters inherently derives from the information content they can exploit (e.g., Murray, Bennett, \& Sekuler, 2005). For instance, increased viewing distance shifts the bandwidth of spatial frequency information conveyed to the retina (Burt \& Adelson, 1983; Sowden \& 
Schyns, 2006) (Figure 1a), until it falls beyond the retina's contrast sensitivity, at which point face identification performance deteriorates to chance (Ramon, 2015). Crucially, as the spatial frequency content shifts in this way, the identity information (or signal) it conveys is gradually lost following a psychometric function. Psychophysically, the maximum slope of the psychometric function directly relates to the standard deviation of the probability density function: the greater the cumulative distribution's slope, the less variability is present in its corresponding probability distribution (Klein, 2001). Thus, psychophysical assessment of SRs provides a potentially useful window into the consistency with which they exploit stimulus information relative to others, beyond their simple ability (typically measured by the proportion of correct responses alone).

Tardif and colleagues (2019) recently provided the first experimental psychophysical evidence of differential spatial frequency information exploitation in face stimuli between SRs and normals. They found that SRs' superior performance stems from exploitation of the same local facial feature information as normal counterparts (Tardif et al., 2019). However, this could also be explained by more consistent (i.e., less variable) information exploitation among SRs. Moreover, due to the 'Bubbles' response classification method (Gosselin \& Schyns, 2001) used by Tardif et al. (2019), (which involves provision of only piecemeal local featural information) usage of spatial frequency information as would be available to the retina under normal viewing conditions (Sowden \& Schyns, 2006) was not probed. Previous studies with normal observers have shown familiarity-dependent enhancement of selectivity for retinally available horizontal, but not vertical, spatial frequency information across the whole face stimulus (Figure 1 b) for upright identity matching (c.f., Pachai et al., 2017). However, this effect has never been assessed in SRs.

a.
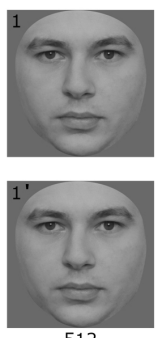

512

b.

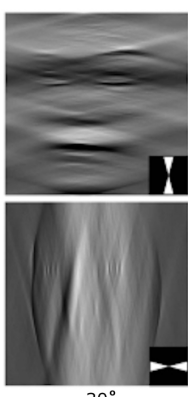

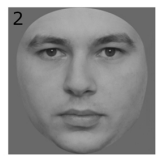

$2^{\prime}$

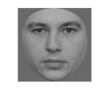

256

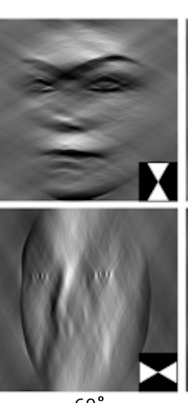

$60^{\circ}$

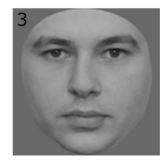

$3^{\prime}$

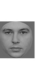

128

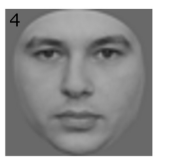

$4^{\prime}$

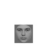

64

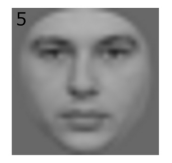

$5^{\prime}$

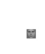

32

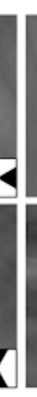

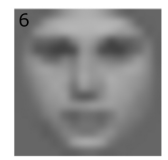

$6^{\prime}$

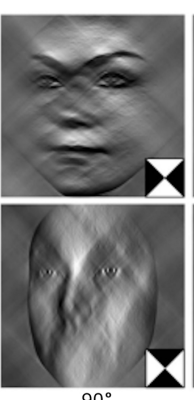

$90^{\circ}$

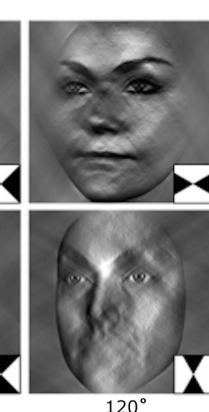

$120^{\circ}$

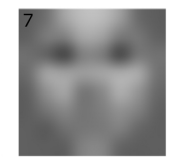

$7^{\prime}$

16

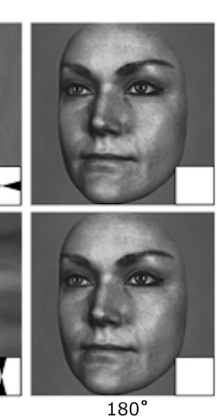

Figure 1. Examples of stimuli used in Experiments 1 and 2. a. In Experiment 1 image size varied logarithmically from 512 pixels in width/height (images 1 and 1') to 8 pixels (images 7 and 7'). The top row of a. shows the effect of the laplacian pyramid on SF con tent, and the bottom row displays the actual stimulus size. b. In Experiment 2, images were bandpass filtered to preserve horizontal (top row of b.) or vertical (bottom row of b.) information in $15^{\circ}$ steps from $0^{\circ}-180^{\circ}$ (with every second step shown here). Panel b. was reproduced from Pachai et al. (2017).

Therefore, the present study aims to psychometrically assess individual differences in the consistency of full-face spatial frequency and orientation information exploitation, across two experiments. In the first, we test whether consistent exploitation of retinally available spatial frequency information (as in Figure 1a) during unfamiliar face matching differentiates SRs from a sample of normal observers as controls (See Method section for details). In the second, testing the same two groups of observers, we assess whether such (potential) differences 
extend to greater selectivity for horizontal spatial frequency structure as shown in previous research (Goffaux, Poncin \& Schiltz, 2015; Taubert et al., 2016; Goffaux \& Greenwood, 2016; Jacobs et al., 2020) (See Method section for details).

Our goals were to determine whether SRs diagnosed using novel conservative criteria (Ramon, 2021) are more sensitive to retinally available spatial frequency information across orientations, as well as whether they more consistently select identity-diagnostic information, exploiting it to enhance their judgments of facial identity.

\section{Method}

All procedures and protocols were approved by the Ethics Committee of the University of Fribourg (approval number 473) and conducted in accordance with both their guidelines, as well as those set forth in the Declaration of Helsinki. All participants were healthy volunteers, provided informed written consent and were not financially compensated for their participation. Participants. Thirty-one students in the Department of Psychology and the University of Fribourg (21 Females; mean age of 27 years; age range of 20-47 years) participated in two experimental sessions in exchange for course credit. SRs who participated in this study were recruited from a larger cohort of recently reported SR individuals (Ramon, 2021). In short, they were identified as disposing of exceptional face processing ability if they achieved high performance (among the top 5\% of previously tested normative samples; Stacchi et al, 2020; Fysh et al., 2020) on at least two of three challenging tests of face cognition: the YBT (long form; Stacchi et al., 2020; Fysh et al., 2020; Bruck et al., 1991), the FICST (Stacchi et al., 2020; Fysh et al., 2020; Jenkins, White, Van Montfort \& Burton, 2011), and the CFMT+ (Russell et al., 2009). SR status was conferred only for those whose performance exceeded the 95\% Cl for typical performance on at least two of the three. No participants in either group were personally familiar with any of the identities of the face stimuli presented throughout the experimental sessions, and all were eye disease-free, with normal or corrected-to-normal visual acuity, as assessed by qCSF (Lesmes, et al., 2010; Canare, Lu \& Ni, 2019; see Supplemental Material, Table 1).

\section{Apparatus}

Psychophysical experiments were designed and implemented using the PsychToolbox (Brainard, 1997; Pelli, 1997; Kleiner et al., 2007) in the Matlab (Mathworks Inc.) programming environment. Two different monitors were beta calibrated and employed for stimulus presentation throughout experimental sessions: a VIEWPixx/3D - 22005C monitor (1980 x 1020 pixel resolution; viewing distance: $68.5 \mathrm{~cm} ; 120 \mathrm{~Hz}$ refresh rate; average luminance of ca. $110 \mathrm{~cd} / \mathrm{m}^{2}$ ), and a Samsung SyncMaster 2233RZ - 3D LCD monitor (1680 x 1050 pixel resolution; viewing distance: $62.2 \mathrm{~cm}$; $60 \mathrm{~Hz}$ refresh rate; average luminance of ca. $110 \mathrm{~cd} /$ m²) (Wang \& Nikolic', 2011).

\section{Procedures}

In both experiments, participants were instructed to complete a 10AFC face matching task as employed e.g. by Pachai et al. (2017), in the context of which on each trial they had to match one initially presented left- or right-facing filtered target identity to one of 10 unfiltered frontfacing potential probe identities. The beginning of each trial was signaled by the appearance of a central fixation point (1s duration), whose disappearance (for 100ms) in turn signaled the appearance of the target identity (250ms duration). This sequence was immediately followed by the aforementioned array of 10 potential probe identities, in two horizontal rows of 5 (with 
males above and females below fixation). Participants were given unlimited time to select one probe that (best) matched the target's, by hovering the mouse cursor over it and pressing the left mouse button to confirm their selection. They were further instructed to prioritize responding correctly over quickly. A matching identity was always presented among the 10 potential probes (i.e., the experiment did not include target-absent trials), while four other same-sex and five opposite-sex identities were randomly drawn from the remaining face identities in the stimulus set to make up the array of potential probes. A fully counterbalanced and randomized sequence of images was presented to each participant. Each session of Experiment 1 consisted of 480 trials, lasting roughly 45 minutes total, including five mandatory break intervals (of participant-determined duration) to alleviate fatigue. All procedures in Experiment 2 were identical, with the exception that the fully counterbalanced and randomized sequence of stimuli comprised 350 trials and 4 break intervals.

\section{Stimuli}

\section{Experiment 1}

Twenty-five male and 25 female faces, sampled from students at Universite catholique de Louvain La Neuve, served as the experimental stimulus set (Laguesse et al., 2012). For each of these face models, three viewpoint images were generated, then converted to grayscale and cropped to exclude external identifying features (e.g. ears, hair) (Adobe Photoshop, Adobe Inc.), centered in a $512 \times 512$ pixels array delimiting all stimuli to $5.4^{\circ}$ of visual angle. One front-facing, one left-rotated and one right-rotated viewpoint were generated per face identity, yielding 60 unique images. Each was then isotropically reduced in scale to each of 7 different pixel sizes (8, 16, 32, 64 and 512 pixels across) as a manipulation of viewing distance, using the Laplacian pyramid model (Burt and Adelson, 1983; Sowden \& Schyns, 2006) to simulate the retinally available information at each size-correspondent viewing distance by effectively removing high spatial frequency content at larger viewing distances (See Figure 1a). All stimuli were finally adjusted to a root-mean-squared contrast of 0.2.

\section{Experiment 2}

Participants were shown the same 10 upright male and 10 upright female identities as used by Pachai et al. (2017), including 3 different viewpoint images of each identity, as in Experiment 1. Here, however, rather than reducing the images' scale as in Experiment 1, we systematically varied the amount of horizontal and vertical information present in these images. All were band-passed using sharp-edged orientation filters centered on $0^{\circ}$ (for horizontal information filtering) or $90^{\circ}$ (for vertical information filtering) (Figure $1 \mathrm{~b}$ ). Then, all were filtered at each of 12 bandwidths in steps of $15^{\circ}\left(15^{\circ}, 30^{\circ}, 45^{\circ}, 60^{\circ}, 75^{\circ}, 90^{\circ}, 105^{\circ}, 120^{\circ}, 135^{\circ}\right.$, $\left.150^{\circ}, 165^{\circ}, 180^{\circ}\right)$, such that $180^{\circ}$ filters passed all horizontal and vertical information (unfiltered stimuli), while $90^{\circ}$ was the largest bandwidth at which horizontal and vertical information was independently isolated (c.f. Pachai et al., 2017).

\section{Results}

Figure 2 displays the psychometric functions across both experiments for the 31 normal observers and 11 SRs. 

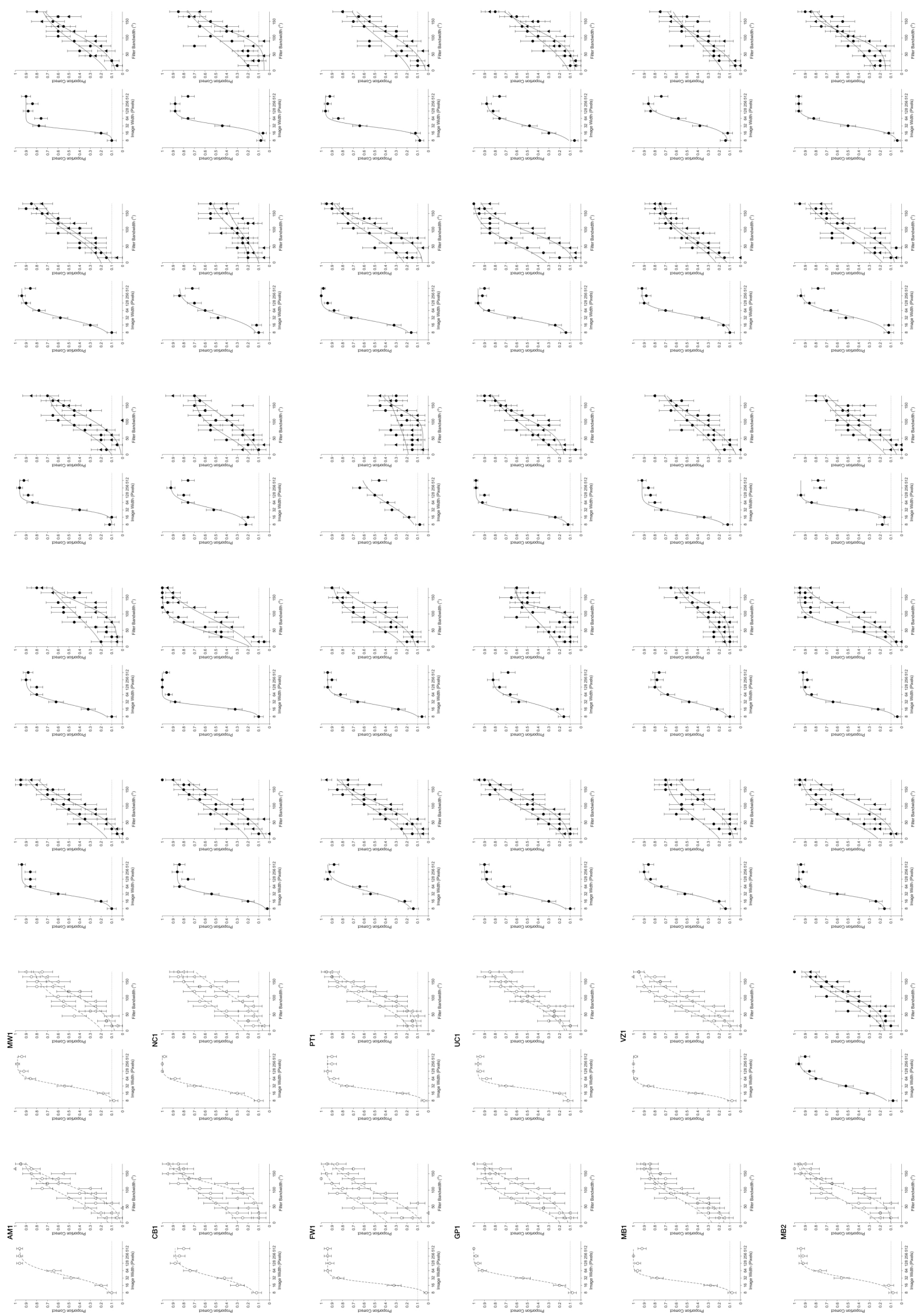

Figure 2. Individual SRs' and normal observers' profiles. Each observer's performance profile is displayed for Experiment 1 and 2 in their respective left and right panels. The 11 SRs are plotted using white markers with dashed lines; normal observers with black markers and solid lines. For Experiment 2, horizontal and vertical structure are plotted with circles and triangles, respectively. Individual SRs' are referred to using their unique acronyms as provided with their original SRdiagnostic information by Ramon (in press). In each plot, chance performance for 10AFC (0.1 proportion correct responding) is denoted by a dotted line. Error bars represent \pm 1 SEM for each data point. 


\section{Experiment 1}

To determine the extent to which SRs and normal observers extract retinally-available spatial frequency information at different simulated viewing distances, we conducted a mixed Group (2, between) x Image Size (7, within) ANOVA on observers' performance. We found significant main effects of both Group $(F(1,40)=6.84, p=.014)$ and Image Size $(F(6,40)=749.9$, $p<.0001)$, as well as a significant Group by Image Size interaction $(F(6,240)=2.91, p=.009)$.

In order to characterize the interaction between Group and Image Size, we conducted a set of FDR-corrected (false discovery $a=.05$ ) independent samples t-tests on SRs' versus normal observers' performance at every image size condition, each one testing the alternative hypothesis that SRs' performance differs from normal observers'. We reject the null hypothesis of equal performance between Groups at Image Sizes from 64-256 pixels (t(40)=-1.91, 1.33, $1.95,2.33^{\star}, 2.77^{\star}, 2.66^{\star}, 2.43$; asterisked t-values indicate $p<a_{F D R}$ ), implying that SRs outperformed normal observers over the aforementioned range of widths (see Figure 3, bold line). However, this does not on its own distinguish between whether SRs and normal observers exploited retinally available spatial frequency information over different ranges of viewing distances, or whether SRs exhibit enhanced sensitivity relative to normal observers within the same range.

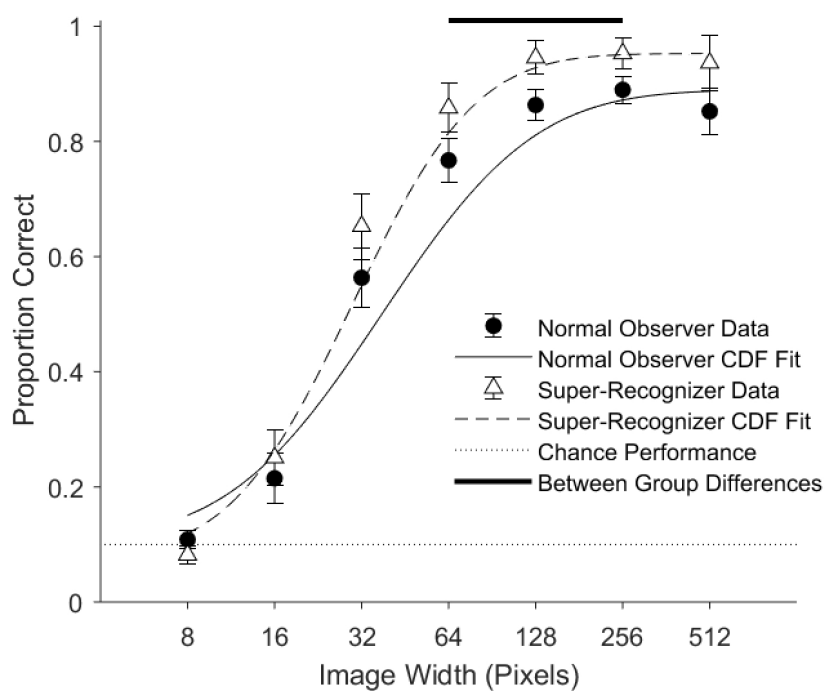

Figure 3. CDF fitted performance for Experiment

1. Data are plotted separately for normal (solid line) and SR (dashed line) observers across image size conditions. Error bars represent \pm 1 SEM; bold line denotes image widths at which SRs significantly outperform normal observers. See Figure 1 for individual observers' profiles.

To determine whether SRs and normal observers exploited retinally available spatial frequency information over the same range of simulated viewing distances, we computed their absolute thresholds as the smallest image size condition for which the 95\% Cl about their proportion of correct responses excluded chance. An independent samples t-test of the difference between thresholds assessed the alternative hypothesis that SRs' absolute thresholds differed from those of normal observer'. We fail to reject the null hypothesis that SRs' absolute thresholds were different from normal observers' $(t(40)=0.51, p=.613$ ) (mean thresholds of $16 \pm 2$ pixels and $16 \pm 3$ pixels, respectively). Thus, we have no evidence that SRs exploit retinally available spatial frequency information over a wider range of image sizes than normal observers.

\section{Experiment 2}

We conducted a mixed Group (2, between) x Filter Orientation (2, within) ANOVA on the mean performance for filter bandwidths between $15^{\circ}$ and $90^{\circ}$ (c.f. Pachai et al., 2017). First, we found a significant main effect of Group $(F(1,40)=5.31, p=.026)$, indicating that SRs 
outperformed normal observers. Second, we found a main effect of Filter Orientation $(F(1,40)=190.64, p<.0001)$, indicating that performance was greater for images where horizontal information was retained than vertical. Finally, we found a marginal trend towards a Group by Filter Orientation interaction $(F(1,1)=2.85, p=.099$ ) (see Figure 4 and Figure $5 a$ for descriptive statistics). An ANOVA conducted for the bandwidths where horizontal and vertical orientation information were independently presented (i.e. bandwidths below 90) yielded similar results (see Supplemental Material, Table 2).
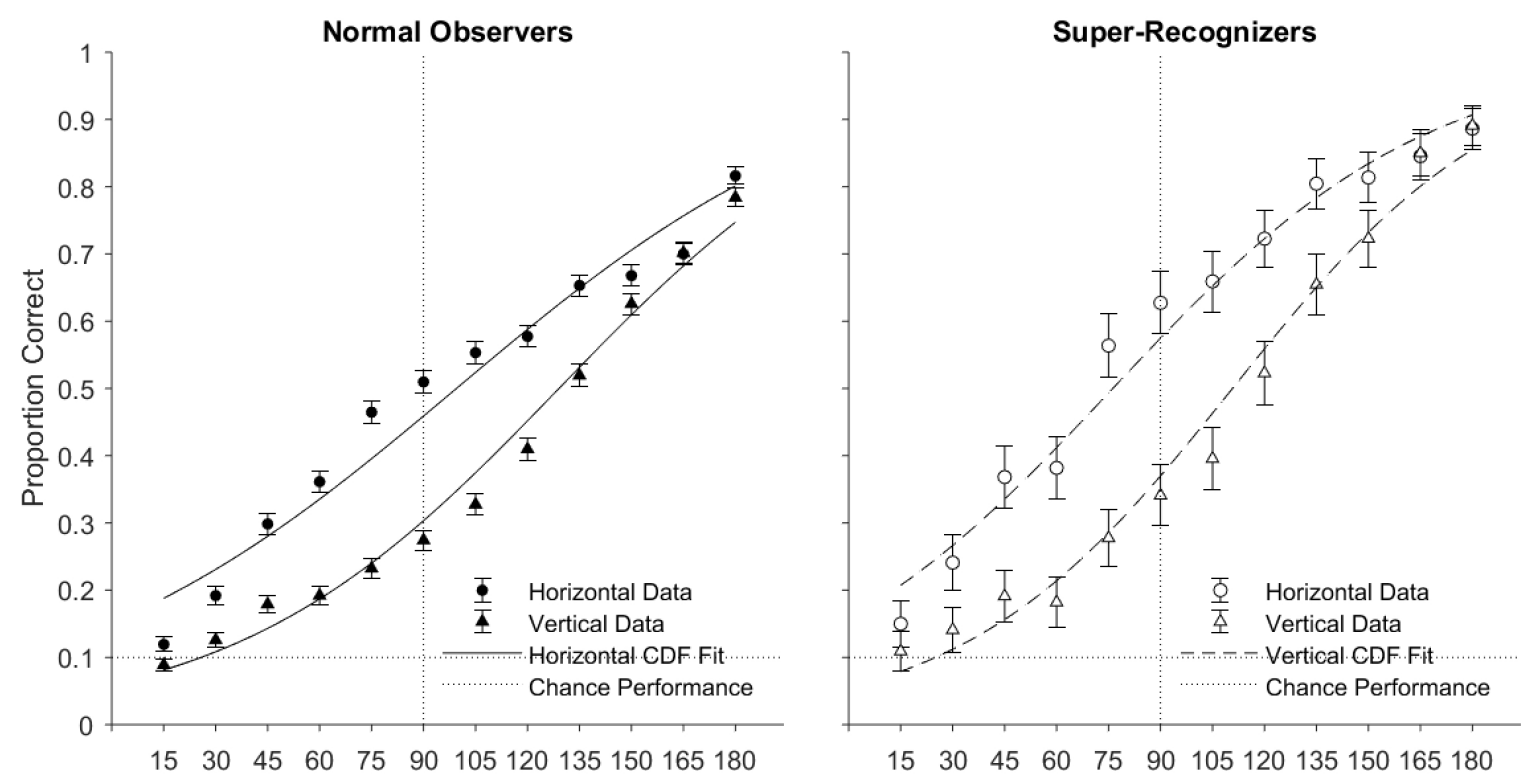

Filter Bandwidth $\left({ }^{\circ}\right)$

Figure 4. Comparison of CDF fits to observed performance in Experiment 2. Performance exhibited by normal observers (left) and SRs (right); error bars denote \pm 1 SEM for each data point. Vertical dotted lines denote $90^{\circ}$ bandpass filter orientation (the largest bandwidth at which horizontal and vertical SF information remain isolated from one another); horizontal dotted lines denote chance performance for the 10AFC task (0.1 proportion correct responses).

To establish selectivity profiles in both groups, we conducted a series of 24 planned, FDRcorrected (false discovery $a=.05$ ), independent samples t-tests of horizontal selectivity. Twelve such tests were conducted for normal observers, $\left(t(30)=1.99,3.51^{*}, 5.00^{*}, 8.37^{\star}, 9.27^{*}\right.$, $8.60^{*}, 7.53^{*}, 8.00^{*}, 6.07^{*}, 1.59,-0.09,1.70$; asterisked t-values indicate $p<a_{F D R}$ ) and 12 for SRs $\left(t(10)=1.69,2.80^{*}, 3.11^{\star}, 4.75^{\star}, 9.72^{*}, 7.62^{*}, 6.42^{*}, 3.93^{*}, 3.87^{\star}, 1.74,-0.11,-0.15\right.$; asterisked t-values indicate $\left.\mathrm{p}<\mathrm{a}_{\mathrm{FDR}}\right)$. Each t-test evaluated the alternative hypothesis that selectivity differed significantly from 0 . We reject the null hypothesis of no selectivity from $30^{\circ}$ to $135^{\circ}$ in both groups, and fail to reject it at all other bandwidths (Figure 5). From this we gather that SRs and normal observers display similar ranges of horizontal selectivity, but not whether this differs between groups at any specific bandwidth. In other words, these findings address the first of our two overarching hypotheses - that SRs might display selectivity at different bandwidths than normal observers - and we find no supporting evidence for this. However, it does not address the second, concerning whether SRs might demonstrate higher selectivity within any particular bandwidth(s). 
a.

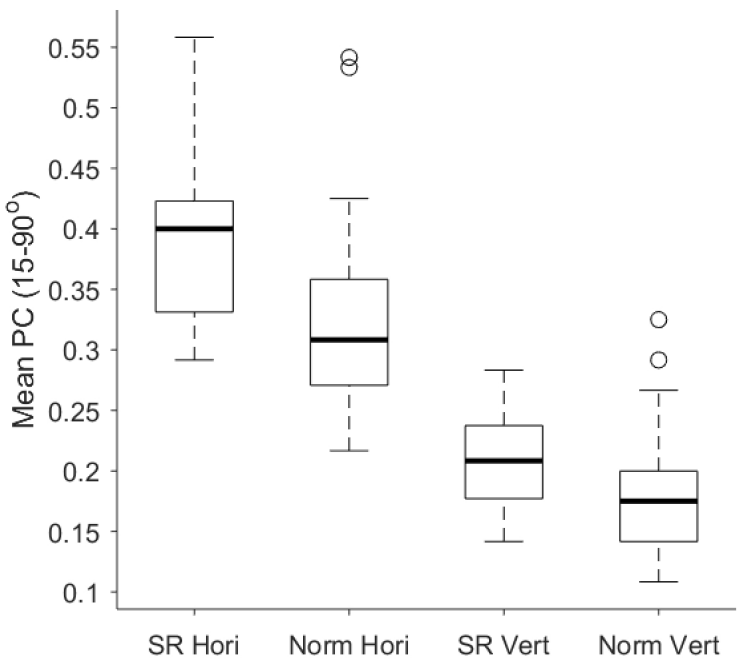

b. $\quad 0.4-\longrightarrow$

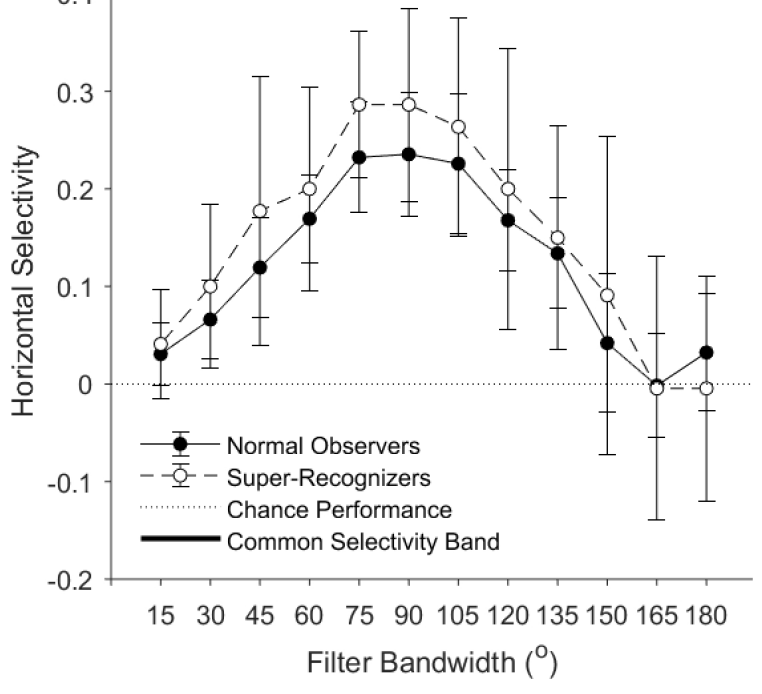

Figure 5. Visualizations of Group by Filter Orientation differences. a. Box and whisker plots of horizontal selectivity for the average proportion correct obtained from $15^{\circ}-90^{\circ}$ filtered target stimuli. b. Plot of horizontal selectivity (difference in performance between horizontal and vertical filter orientations) for normal observers (solid line) and SRs (dashed line). Error bars represent the FDR-corrected 95\% Cl about the mean at each data point; the bold horizontal line above the groups' selectivity functions indicates bandwidths at which normal observers and SRs displayed significant horizontal selectivity.

Therefore, in order to test whether SRs might show greater selectivity than normal observers within any particular bandwidth, we conducted a series of 12 planned, FDR-corrected (false discovery $\mathrm{a}=.05)$, independent samples t-tests of SRs' versus normal observers' selectivity to test the alternative hypothesis that SRs' horizontal selectivity differs from normals' at each filter bandwidth (i.e., between-groups tests of the within-observer difference between horizontal and vertical performance). We reject the null hypothesis that SRs and normal observers have comparable horizontal selectivity at bandwidths from $30^{\circ}-45^{\circ}$, and $75^{\circ}-90^{\circ}(\mathrm{t}(40)=0.93$, $2.51^{\star}, 3.39^{\star}, 2.12,3.02^{\star}, 2.60^{\star}, 1.77,2.15,1.02,2.61,-0.22,-2.70$; asterisked t-values indicate $\mathrm{p}<\mathrm{a}_{\mathrm{FDR}}$ ), and fail to reject it elsewhere. Overall, this implies that SRs and normal observers might show slightly differential selectivity at the aforementioned bandwidths, though we caution that the observed interaction effect is marginal.

\section{Individual Differences in Consistency Across Experiments}

Experiments 1 and 2 aimed to test the hypotheses that SRs are either able to exploit a wider range of information during face-matching than normal counterparts, or simply more sensitive to spatial frequency information within the same range as normal counterparts during facematching. Individually, each experiment supports the latter.

With this in mind, we sought to classify SRs based on psychophysical performance. Since the psychometric function describes the extent to which increased stimulus information provides useful evidence towards correct judgments, the steeper the increase in performance with stimulus strength, the more consistently that information is incorporated in decisions. Therefore, in order to quantify observers' consistency, we computed the maximum slope (at the inflection point of the psychometric function) of each observer's fitted psychometric functions separately (Figure 6), first across spatial frequency bandwidths (Experiment 1), then for horizontal bandpass filter orientations, and finally for vertical bandpass filter orientations (Experiment 2). 


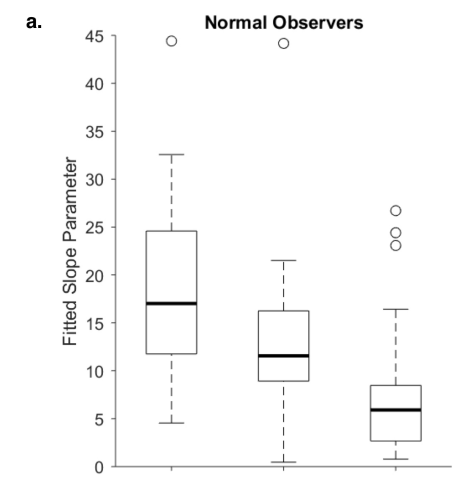

b.

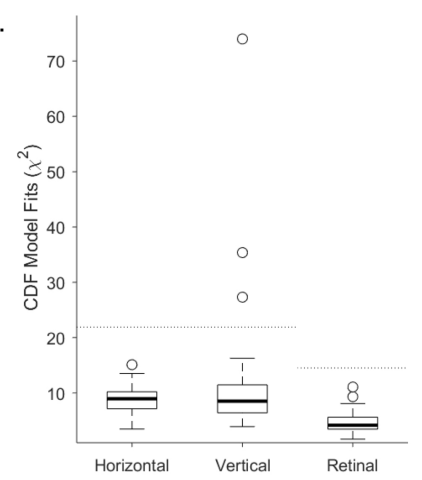

Super-Recognizers

○
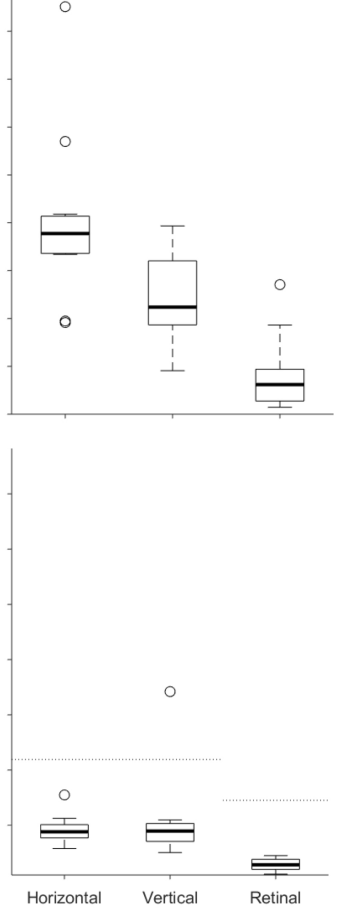

Figure 6. Individual differences in slope and $\chi^{2}$ fitcross Experiments 1 and 2. a. Normal observers' and SRs' slopes taken from individually fitted data to CDFs with two parameters: slope (shown here) and PSE. b. Individual Chi Square fit values for CDFs across both experiments for normal observers and SRs; dotted lines denote critical values of $\chi 2$ (for $\boldsymbol{\alpha}=.05$ )

Of course, an observer's slope is only a useful metric of their consistency insofar as the psychometric function actually describes their performance. So, we computed $\chi^{2}$ fits (Figure 7) to the psychometric functions obtained in Experiments 1 and 2 (c.f. Figures 3 and 4), each testing the null hypothesis that the obtained data came from the population described by the theoretical curve to which they were fitted. Among normal observers, only 3 of the 93 fits (31 observers, 3 fits each) reached statistical significance (leading us to reject the null hypothesis). Among SRs, only 1 of the 33 fits (11 observers, 3 fits each) reached significance.

Finally, we conducted a k-means cluster analysis (constrained to two groups) on observers' slope parameters across both experiments. This procedure iteratively classifies observers into k clusters, by minimizing the euclidean distance from assigned cluster centroids. Here, we used it to examine whether psychometric slope would be diagnostic of superior performance (Figure 7). Although we do find a positive point-biserial correlation between group and psychometric slope in Experiment $1(\mathrm{r}(40)=-.39, \mathrm{p}=.011)$, the cluster analysis yielded low sensitivity (classification $\left.d^{\prime}=.25\right)$. Moreover, neither horizontal $(r(40)=-.08, p=.615)$, nor vertical $(r(40)=-.10, p=.529)$ filter orientation conditions in Experiment 2 predict SR status. 


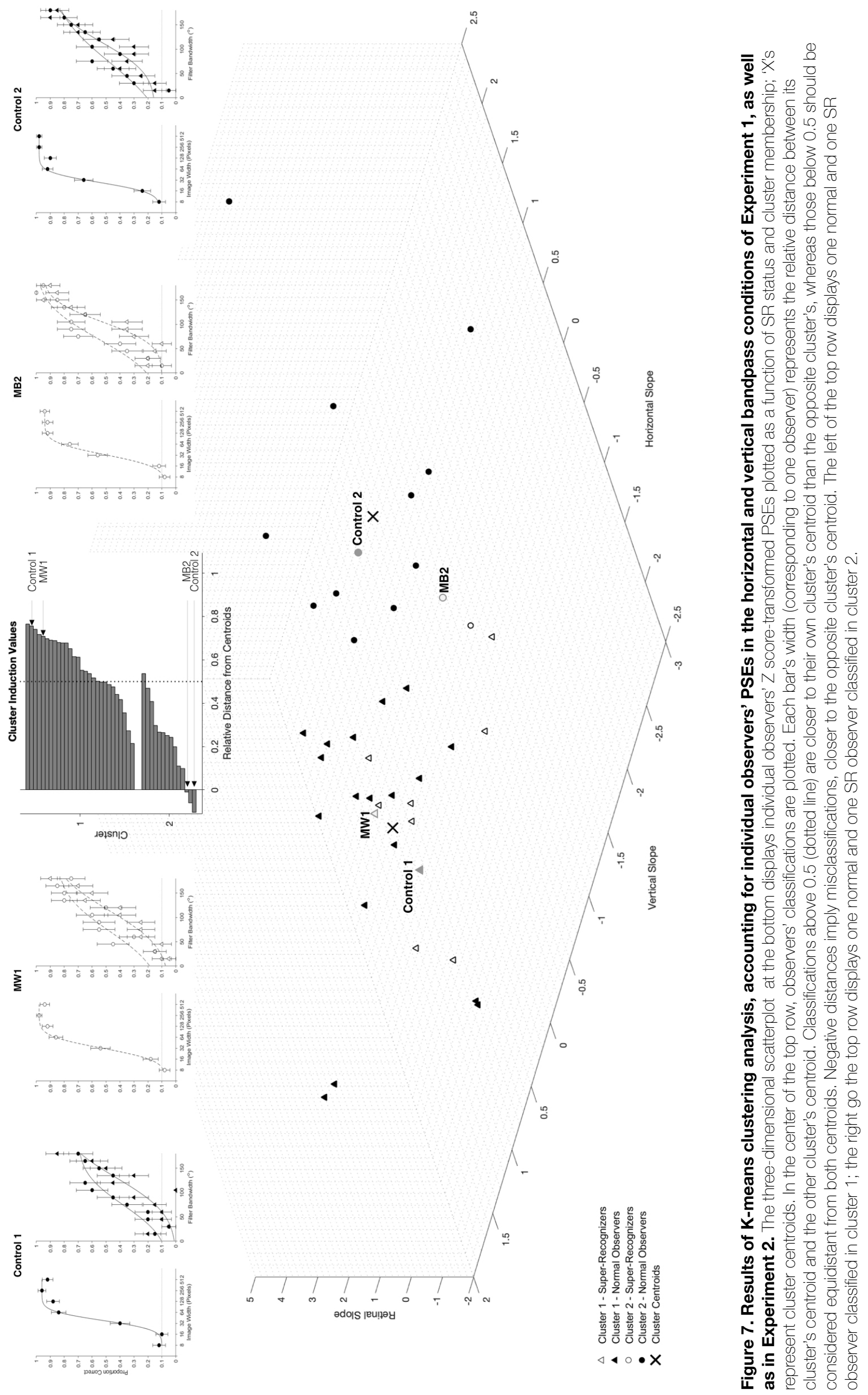




\section{Discussion}

In Experiment 1, SRs more efficiently employed retinally available spatial frequency information in face matching over the same range of simulated viewing distances as their normal counterparts, while significantly outperforming them at larger image sizes (i.e. smaller viewing distances; see Figure 3). Thus, in terms of information usage, it seems that any performance differences between the two groups are quantitative, in that relative to controls, SRs show no evidence of sensitivity to different spatial frequency spectra (Figure 3). This suggests that they differ only in the consistency with which they exploit retinally available spatial frequency information. As viewing distance increases, spatial frequency content of the original image shifts to lower bands. Relative to their normal counterparts, SRs show steeper psychometric slopes, and thus more consistently exploit this information, even though both groups are indeed sensitive to it (i.e., are above threshold) within the same range of spatial frequencies.

Previous research (Tardiff et al., 2019) has assessed SRs' performance using the 'Bubbles' response classification method (Gosselin \& Schyns, 2001; see also Haig, 1984). This method applies random spatial frequency bands to local features of faces only. Therefore, observers' exploitation of spatial frequency information under this particular circumstance differs from that which, according to Sowden \& Schyns (2006), would be found under normal viewing conditions. While offering important insights into the contributions of different spatial frequency bands to identification of particular facial features/locations, the Bubbles technique cannot determine the identity-diagnostic information content conveyed by a whole face stimulus. Regardless, as concerns information exploitation, using full face information manipulations, we also conclude that SRs and normal observers utilize roughly the same range of information. However, here we add that SRs exploit this information more consistently, and under more naturalistic conditions of information availability.

In our second experiment, we find horizontal selectivity commensurate with previous studies (Goffaux, Poncin \& Schiltz, 2015; Goffaux \& Greenwood, 2016; Pachai et al, 2017; Jacobs et al., 2020). Specifically, horizontal spatial frequency information is more diagnostic for facial identity matching than vertical, among both groups of observers. SRs significantly outperformed normal observers - across filter bandwidths and orientations - suggesting that they have higher sensitivity to spatial frequency content when matching facial identity. But critically, in terms of our initial hypotheses, it seems that there is at best modest evidence that SRs display differential horizontal selectivity relative to their normal counterparts. While we observed a marginal interaction between Group and Filter Orientation, we find no evidence in our planned comparisons that SRs employ qualitatively different spatial frequency bands than normal observers (see Figure 2 for individual observers' profiles). Between group differences in horizontal selectivity (if any) seem to relate to quantitative differences in information exploitation within a spatial frequency band common to both groups: $30^{\circ}-45^{\circ}$, and $60^{\circ}-75^{\circ}$. In sum, while we do find evidence of horizontal selectivity commensurate with previous research, it is not particularly diagnostic of SR status.

Of note, though, the between-group differences observed in Experiment 1 were only found for image sizes smaller than those employed in Experiment 2. This could have impaired our ability to measure between-group differences in spatial frequency or orientation information exploitation at the spatial scales considered here. Consequently, the relatively small Group by Filter Orientation interaction might result in part from the larger image sizes used in Experiment 2. Over a wider range of simulated viewing distances, with covaried orientation information 
filtering, it could be possible to assess the contributions of orientation information to super recognizers' performance relative to normal controls. However, such a manipulation is beyond the scope of the current study.

Considering the individual differences across experiments (c.f. Figures 2, 6, and 7), it seems that SRs' superior face processing abilities stem from their capacity to exploit a common band of spatial frequency information more consistently than normal observers. While both groups show comparable absolute thresholds for retinally available spatial frequency content across spatial scales, as well as for horizontally and vertically oriented spatial frequency content, we find that consistency of retinally available spatial frequency exploitation (i.e. psychometric slope) predicts SR status, irrespective of orientation. However, cluster analyses suggest that within-observer consistency (i.e., psychometric function slope) has low overall diagnostic value (Figure 7), in that more than half of normal observers were classified as belonging to the same cluster as SRs. Overall, we surmise that within-observer consistency constitutes an important aspect of SRs' performance, in that it is predictive of their status, but is not alone sufficient to diagnose them as such.

We therefore propose that future studies aiming to classify SRs ought to adopt measures that are optimized to assess the consistency with which observers exploit identity-diagnostic information for face cognition, in addition to more traditional performance metrics. This would address the expressed need for appropriate diagnostic tools and prevention of false positive classifications of superior face processing ability (Ramon et al., 2019; Devue, 2019; Moreton, Pike \& Harvard, 2019; Robertson \& Bindemann, 2019). Unfortunately, SR diagnostic tests often employ only simple performance criteria, even though Russell and colleagues' (2009) seminal study of SRs adopted criteria based on tests of three separate subprocesses (i.e., perceptual matching, old/new recognition and identification). Recent studies have nevertheless adopted less stringent criteria, often testing only a single subprocess (Bobak et al., 2016a; Bobak, Pampoulov \& Bate, 2016; Bobak, Dowsett \& Bate, 2016; Phillips et al., 2018), or using measures designed to diagnose DP (Tardiff et al., 2019). Minimally, though, multiple sufficiently sensitive measures should be applied in the diagnostic procedure to ensure that evidence of superior performance within an individual is at least consistently superior across tasks/subprocesses. Moreover, in light of the present results, systematic parametric variations in the stimuli they employ should be applied to existing tests, as these would provide additional diagnostic information. We recognize that this can be a cumbersome procedure, and therefore propose that future SR testing materials include assessments of observers' consistency within-task, by measuring the resulting psychometric function's slope.

Given that SRs did not show lower absolute thresholds than controls during unfamiliar facial identity matching-either at large viewing distances, or at narrow spatial frequency bandwidths - it could be that identity learning depends on identity diagnostic spatial frequency content. Considering that Pachai et al. (2017) reported enhanced horizontal selectivity at narrower bandwidths for familiar relative to unfamiliar faces, comparison of normals and SRs for familiar and unfamiliar face recognition under otherwise similar conditions could be instrumental to understanding the informational basis of familiarity formation for (a given) facial identity. Pachai and colleagues' (2017) previous study employed the same experimental procedures as our second experiment, but any exposure-related familiarity developed over the course of the experiment was insufficient to mask group differences related to real-life, acquired, personal familiarity. Interestingly, they compared two groups of observers who were 
either personally familiar or not with the identities depicted throughout the experiment. They found greater horizontal selectivity among those who were familiar with the face stimuli than not. Thus, if SRs were indeed different from normal controls in terms of quickly building familiarity with new identities, we would have expected to see increased horizontal selectivity among them, yet we do not.

The fact that SRs outperform normal observers for retinally available spatial frequency information, but only at larger viewing distances, warrants an investigation into selectivity for information at different spatial scales. Future work ought to consider whether different kinds of information are utilized (say, at different spatial scales) when matching familiar and unfamiliar faces. Particularly, concomitant systematic variation of both orientation and retinal available spatial frequency information would be instrumental in disentangling the effects observed in the presently discussed experiments.

\section{Conclusion}

To summarize, we tested proficiency for matching of unfamiliar facial identity in a group of 11 conservatively diagnosed SRs and 31 normal observers. Findings from two experiments involving uniform parametric manipulations of spatial frequency content and orientation across the full face suggest that the difference between SRs and normal observers is of quantitative, not qualitative, nature. Therefore, based on the currently available psychophysical findings, SRs cannot be described as a special class (Young \& Noyes, 2019), but rather as experts among experts. Rather than accepting this notion, we emphasize that further work using complementary methods is needed to ascertain whether SRs process facial information in a purely quantitatively different manner. Indeed, it is plausible that qualitative differencessimilarly to those reported for developmental prosopagnosia (Behrmann, Scherf \& Avidan, 2016) - may be found in large cohorts of carefully described SR cases identified using the same stringent diagnostic framework (Ramon, 2021).

\section{Author contributions}

MR \& MVP designed the experiments; MZ acquired the data; JDN analyzed the data; MR and JDN wrote the manuscript.

\section{Acknowledgements}

MR is supported by a Swiss National Science Foundation PRIMA (Promoting Women in Academia) grant (PROOP1_179872).

\section{Conflict of interest statement}

The authors declare no conflict of interest.

\section{Data Accessibility Statement}

Accompanying data can be downloaded from the Open Science Framework (https://ospatial frequency.io/x2ksa/).

\section{References}

Bate, S., Frowd, C., Bennetts, R., Hasshim, N., Murray, E., Bobak, A. K., Wills, H., \& Richards, S. (2018). Applied screening tests for the detection of superior face recognition. Cognitive Research: Principles and Implications, 3(22), 19. https://doi.org/ 41235-018-0116-5 
Bobak, A. K., Dowsett, A. J., \& Bate, S. (2016). Solving the Border Control Problem: Evidence of Enhanced Face Matching in Individuals with Extraordinary Face Recognition Skills. 1-13. https://doi. org/10.1371/journal.pone.0148148

Behrmann, M., Scherf, K. S., \& Avidan, G. (2016). Neural mechanisms of face perception, their emergence over development, and their breakdown. Wiley Interdisciplinary Reviews. Cognitive Science, 7(4), 247-263. https://doi. org/10.1002/wcs.1388

Bobak, A. K., Hancock, P. J. B., \& Bate, S. (2016). Super-recognisers in Action: Evidence from Face-matching and Face Memory Tasks. 91 (October 2015), 81-91. https://doi.org/ 10.1002/acp.3170

Bobak, A. K., Pampoulov, P., \& Bate, S. (2016). Detecting superior face recognition skills in a large sample of young British adults. Frontiers in Psychology, 7(SEP), 1-11. https:// doi. org/10.3389/fpsyg. 2016.01378

Bobak, A. K., Parris, B. A., Gregory, N. J., Bennetts, R. J., \& Bate, S. (2017). Eye-movement strategies in developmental prosopagnosia and "super" face recognition. Quarterly Journal of Experimental Psychology, 70(2), 201-217. https://doi.org/ $\underline{10.1080 / 17470218.2016 .1161059}$

Burt, P. J., \& Adelson, E. H. (2009). The Laplacian pyramid as a compact image code. Fundamental Papers in Wavelet Theory, C(4), 28-36. https://doi.org/ $10.1515 / 9781400827268.28$

Brainard, D. H. (1997). The psychophysics toolbox. Spatial vision, 10(4), 433-436.

Bruck, M., Cavanagh, P., \& Ceci, S. J. (1991). Fortysomething: Recognizing faces at one's 25th reunion. Memory \& Cognition, 19(3), 221-228. https://doi.org/10.3758/ BF03211146

Canare, D., Ni, R., \& Lu, T. (2019). An open-source implementation of the Quick Cspatial frequency method. Journal of Vision, 19(10), 86b-86b.

Devue, C., Wride, A., \& Grimshaw, G. M. (2019). New insights on real-world human face recognition. Journal of Experimental Psychology: General, 148(6), 994-1007. https:// doi. org/10.1037/xge0000493

Duchaine, B., \& Nakayama, K. (2006). The Cambridge Face Memory Test: Results for neurologically intact individuals and an investigation of its validity using inverted face stimuli and prosopagnosic participants. Neuropsychologia, 44(4), 576-585. https://doi.org/ 10.1016/i.neuropsychologia.2005.07.001

Duchaine, B., \& Nakayama, K. (2005). Dissociations of face and object recognition in developmental prosopagnosia. Journal of Cognitive Neuroscience, 17(2), 249-261. https://doi.org/10.1162/0898929053124857

Duchaine, B. C., Yovel, G., Butterworth, E. J., \& Nakayama, K. (2006). Prosopagnosia as an impairment to face-specific mechanisms: Elimination of the alternative hypotheses in a developmental case. Cognitive Neuropsychology, 23(5), 714-747. https://doi.org/ 10.1080/02643290500441296

Estudillo, A. J., \& Bindemann, M. (2014). Generalization across view in face memory and face matching. I-Perception, 5(7), 589-601. https://doi.org/10.1068/i0669 
Fox, E., \& Bindemann, M. (2020). Individual differences in visual acuity and face matching ability. Applied Cognitive Psychology, 34(5), 1034-1046. https://doi.org/10.1002/ acp.3682

Fysh, M. C. (2018). Individual differences in the detection, matching and memory of faces. Cognitive Research: Principles and Implications, 3(1). https://doi.org/10.1186/ s41235-018-0111-X

Fysh, M. C., \& Bindemann, M. (2017). Forensic face matching: A review. Face Processing: Systems, Disorders and Cultural Differences, 1-20.

Fysh, M. C., Stacchi, L., \& Ramon, M. (2020). Differences between and within individuals, and subprocesses of face cognition: implications for theory, research and personnel

selection. Royal Society Open Science, 7(9), 200233. https://doi. org/10.1098/rsos.200233

Goffaux, V., \& Greenwood, J. A. (2016). The orientation selectivity of face identification. Scientific Reports, 6. https://doi.org/10.1038/super-recognizerep34204

Goffaux, V., Jemel, B., Jacques, C., Rossion, B., \& Schyns, P. G. (2003). ERP evidence for task modulations on face perceptual processing at different spatial scales. Cognitive Science, 27(2), 313-325. https://doi.org/10.1016/S0364-0213(03)00002-8

Goffaux, V., Poncin, A., \& Schiltz, C. (2015). Selectivity of face perception to horizontal information over lifespan (from 6 to 74 Year Old). PLoS ONE, 10(9), 1-17. https://doi.org/ 10.1371/journal. pone.0138812

Gosselin, F., \& Schyns, P. G. (2001). Bubbles: A technique to reveal the use of information in recognition tasks. Vision Research, 41(17), 2261-2271. https://doi.org/10.1016/ S0042-6989(01)00097-9

Haig N., D. (1985). How Faces Differ - A New Comparative Technique. Perception, 14(5), 601-615. https://doi.org/10.1068/p140601

Klein, S. A. (2001). Perception \& Psychophysics, 63(8), 1421-1455. https://doi.org/https:// doi.org/10.3758/BF03194552

Jacobs, C., Petras, K., Moors, P., \& Goffaux, V. (2020). Contrast versus identity encoding in the face image follow distinct orientation selectivity profiles. PLoS ONE, 15(3), 1-22. https://doi. org/10.1371/journal.pone.0229185

Jenkins, R., White, D., Van Montfort, X., \& Mike Burton, A. (2011). Variability in photos of the same face. Cognition, 121(3), 313-323. https://doi.org/10.1016/i.cognition.2011.08.001

Kleiner, M., Brainard, D., Pelli, D., Ingling, A., Murray, R., \& Broussard, C. What's new in Psychtoolbox-3?

Laguesse, R., Dormal, G., Biervoye, A., Kuefner, D., \& Rossion, B. (2012). Extensive visual training in adulthood significantly reduces the face inversion effect. Journal of Vision, 12(10), 1-13. https://doi.org/10.1167/12.10.14

Lander, K., Bruce, V., \& Bindemann, M. (2018). Use-inspired basic research on individual differences in face identification: implications for criminal investigation and security. 1-13. https://doi.org/10.1186/s41235-018-0115-6

Lesmes, L. A., Lu, Z. L., Baek, J., \& Albright, T. D. (2010). Bayesian adaptive estimation of the contrast sensitivity function: The quick Cspatial frequency method. Journal of Vision, 10(3), 1-21. https://doi.org/10.1167/10.3.17 
Moreton, R., Pike, G., \& Havard, C. (2019). A task- and role-based perspective on superrecognizers: Commentary on 'Super-recognizers: From the lab to the world and back again.' British Journal of Psychology, 110(3), 486-488. https://doi.org/10.1111/ bjop. 12394

Murray, R. F., Bennett, P. J., \& Sekuler, A. B. (2005). Classification images predict absolute efficiency. Journal of Vision, 5(2), 139-149. https://doi.org/10.1167/5.2.5

Pachai, M. V., Sekuler, A. B., Bennett, P. J., Schyns, P. G., \& Ramon, M. (2017). Personal familiarity enhances sensitivity to horizontal structure during processing of face identity. Journal of Vision, 17(6), 1-11. https://doi.org/10.1167/17.6.5

Pelli, D. G. (1997). The VideoToolbox software for visual psychophysics: Transpatial frequencyorming numbers into movies. Spatial vision, 10(4), 437-442.

Phillips, P. J., Yates, A. N., Hu, Y., Hahn, C. A., Noyes, E., Jackson, K., \& Cavazos, J. G. (2018). Face recognition accuracy of forensic examiners, superrecognizers, and face recognition algorithms. 115(24), 6171-6176. https://doi.org/10.1073/pnas.1721355115

Ramon, M. (2015). Perception of global facial geometry is modulated through experience. https://doi.org/10.7717/peeri.850

Ramon M (2021). Super-Recognizers - a novel diagnostic framework, 40 cases, and guidelines for future work. Neuropsychologia

Ramon, M., Bobak, A. K., \& White, D. (2019). Super-recognizers: From the lab to the world and back again. British Journal of Psychology, 110(3), 461-479. https://doi.org/10.1111/ bjop. 12368

Robertson, D. J., \& Bindemann, M. (2019). Consolidation, wider reflection, and policy: Response to 'Super-recognisers: From the lab to the world and back again.' British Journal of Psychology, 110(3), 489-491. https://doi. org/10.1111/bjop.12393

Russell, R., Duchaine, B., \& Nakayama, K. (2009). Super-recognizers: People with extraordinary face recognition ability. Psychonomic Bulletin and Review, 16(2), 252-257. https://doi.org/10.3758/PBR.16.2.252

Stacchi, L., Huguenin-Elie, E., Caldara, R., \& Ramon, M. (2020). Normative data for two challenging tests of face matching under ecological conditions. Cognitive Research: Principles and Implications, 5(1). https://doi.org/10.1186/s41235-019-0205-0

Schyns, P. G. (1998). Diagnostic recognition: Task constraints, object information, and their interactions. Cognition, 67(1-2), 147-179. https://doi.org/10.1016/ S0010-0277(98)00016-X

Sowden, P. T., \& Schyns, P. G. (2006). Channel surfing in the visual brain. Trends in Cognitive Sciences, 10(12), 538-545. https://doi.org/10.1016/j.tics.2006.10.007

Tardif, J., Morin Duchesne, X., Cohan, S., Royer, J., Blais, C., Fiset, D., Duchaine, B., \& Gosselin, F. (2019). Use of Face Information Varies Systematically From Developmental Prosopagnosics to Super-Recognizers. Psychological Science, 30(2), 300-308. https:// doi.org/10.1177/0956797618811338

Wang, P., \& Nikolić, D. (2011). An LCD monitor with sufficiently precise timing for research in vision. Frontiers in Human Neuroscience, 5(AUGUST), 1-10. https://doi.org/10.3389/ fnhum.2011.00085 
White, D., Rivolta, D., Burton, A. M., Al-Janabi, S., \& Palermo, R. (2017). Face matching impairment in developmental prosopagnosia. Quarterly Journal of Experimental Psychology, 70(2), 287-297. https://doi.org/10.1080/17470218.2016.1173076

Young, A. W., \& Noyes, E. (2019). We need to talk about super-recognizers Invited commentary on: Ramon, M., Bobak, A. K., \& White, D. Super-recognizers: From the lab to the world and back again. British Journal of Psychology. British Journal of Psychology, 110(3), 492-494. https://doi.org/10.1111/bjop.12395 


\section{Supplemental Material}

Table 1. Demographic information and results of Quick Contrast Sensitivity (qCSF) assessment. Note that data for two normal and four Super-Recognizers (SRs) are missing as we terminated inclusion of qCSF measurement after t-tests between observer groups performed on data from 37 observers revealed no indication of differences for qCSF parameters.

The only significant group difference that emerged was for observers' age $(t(42)=8.09, p<.01)$.

\section{Observer Demographics}

\begin{tabular}{|l|c|l|l|c|c|c|c|}
\hline Observer ID & $\begin{array}{c}\text { Gend } \\
\text { er }\end{array}$ & Handedness & Age & $\begin{array}{c}\text { Peak } \\
\text { Sensitivity }\end{array}$ & $\begin{array}{c}\text { Peak } \\
\text { Frequency }\end{array}$ & $\begin{array}{c}\text { Bandwidt } \\
h\end{array}$ & CSF Area
\end{tabular}

\section{Normal Observers}

\begin{tabular}{|c|c|c|c|c|c|c|c|}
\hline C1 & $F$ & Right & 27 & 47.27 & 5.00 & 7.35 & 87.10 \\
\hline C2 & $M$ & Right & 27 & 39.14 & 2.26 & 4.62 & 22.53 \\
\hline C3 & $F$ & Right & 21 & 42.21 & 2.11 & 6.58 & 30.84 \\
\hline C4 & $F$ & Right & 23 & 15.19 & 1.23 & 5.54 & 8.08 \\
\hline C5 & $F$ & Right & 21 & 17.10 & 8.46 & 6.15 & 65.66 \\
\hline C6 & $F$ & Right & 21 & 29.13 & 4.52 & 7.83 & 61.61 \\
\hline C7 & $\mathrm{F}$ & Left & 22 & 23.81 & 3.50 & 7.20 & 38.42 \\
\hline C8 & $F$ & Right & 22 & 34.02 & 2.52 & 7.82 & 37.74 \\
\hline C9 & $\mathrm{F}$ & Right & 20 & 27.50 & 3.60 & 3.39 & 22.23 \\
\hline C10 & $\mathrm{F}$ & Right & 26 & 18.39 & 6.86 & 6.66 & 59.64 \\
\hline C11 & $\mathrm{F}$ & Right & 22 & 28.83 & 2.60 & 7.65 & 34.31 \\
\hline C12 & $\mathrm{F}$ & Right & 20 & 19.40 & 2.14 & 4.29 & 13.15 \\
\hline C13 & $\mathrm{F}$ & Right & 20 & 35.81 & 2.99 & 7.11 & 42.35 \\
\hline C14 & $\mathrm{F}$ & Right & 24 & 8.69 & 1.01 & 2.50 & 2.45 \\
\hline C15 & $F$ & Right & 27 & 25.52 & 2.80 & 8.35 & 36.65 \\
\hline C16 & $M$ & Right & 22 & 2.02 & 2.41 & 1.14 & 0.42 \\
\hline C17 & $F$ & Right & 20 & 33.97 & 2.38 & 7.01 & 32.27 \\
\hline C18 & $M$ & Right & 24 & 28.98 & 1.79 & 4.81 & 15.75 \\
\hline C19 & $F$ & Right & 22 & 13.43 & 7.26 & 6.69 & 50.62 \\
\hline C20 & $\mathrm{F}$ & Left & 31 & 22.61 & 2.06 & 7.25 & 21.87 \\
\hline C21 & $F$ & Right & 23 & 26.94 & 1.43 & 6.72 & 16.07 \\
\hline C22 & $M$ & Right & 28 & 38.44 & 3.23 & 8.67 & 57.61 \\
\hline C23 & $\mathrm{F}$ & Right & 29 & 29.07 & 5.72 & 6.76 & 68.10 \\
\hline C24 & $\mathrm{F}$ & Right & 23 & 23.35 & 1.59 & 5.42 & 13.42 \\
\hline C25 & $F$ & Right & 21 & 28.18 & 2.42 & 4.52 & 19.94 \\
\hline C26 & $\mathrm{F}$ & Right & 20 & 20.53 & 2.06 & 7.90 & 22.17 \\
\hline C27 & $M$ & Right & 30 & 29.51 & 2.65 & 8.54 & 39.10 \\
\hline C28 & $\mathrm{F}$ & Right & 20 & 40.55 & 1.86 & 8.83 & 35.02 \\
\hline
\end{tabular}




\begin{tabular}{|c|c|c|c|c|c|c|c|}
\hline C29 & $F$ & Right & 20 & 28.68 & 2.68 & 3.47 & 17.81 \\
\hline C30 & $F$ & Right & 21 & 20.50 & 2.03 & 5.74 & 16.50 \\
\hline C31 & $F$ & Right & 21 & - & - & - & - \\
\hline C32 & $M$ & Right & 30 & - & - & - & - \\
\hline $\begin{array}{l}\text { Normal observers' } \\
\text { mean }\end{array}$ & 23.38 & 26.63 & 3.11 & 6.22 & 32.98 & & \\
\hline $\begin{array}{l}\text { Normal observers' } \\
\text { SD }\end{array}$ & 3.45 & 10.08 & 1.83 & 1.91 & 21.16 & & \\
\hline \multicolumn{8}{|c|}{ Super-Recognizers (SRs) } \\
\hline MB2 & $F$ & Left & 45.0 & 23.66 & 2.17 & 8.48 & 27.25 \\
\hline GP1 & $M$ & Right & 47.0 & 29.38 & 1.40 & 6.66 & 16.54 \\
\hline MB1 & $M$ & Right & 34.0 & 27.35 & 2.85 & 8.09 & 38.05 \\
\hline PT1 & $\mathrm{F}$ & Right & 33.0 & 2.10 & 0.82 & 1.73 & 0.24 \\
\hline $\mathrm{NC} 1$ & $F$ & Right & 41.0 & 29.19 & 2.83 & 7.81 & 38.32 \\
\hline VZ1 & $M$ & Right & 24.0 & 37.05 & 2.46 & 8.61 & 42.60 \\
\hline AM1 & $\mathrm{F}$ & Right & 31.0 & 16.01 & 2.71 & 3.13 & 11.61 \\
\hline FW1 & $M$ & Right & 32.0 & - & - & - & - \\
\hline MW1 & $M$ & Right & 43.0 & - & - & - & - \\
\hline UC1 & $M$ & Right & 42.0 & - & - & - & - \\
\hline CB1 & $F$ & Right & 32.0 & - & - & - & - \\
\hline SRs' mean & 36.73 & 24.51 & 2.57 & 6.09 & 27.31 & & \\
\hline SRs' SD & 7.21 & 11.40 & 0.78 & 2.79 & 15.95 & & \\
\hline
\end{tabular}


Table 2. Expanded analysis of variance (ANOVA) in Experiment 2. Factors included Group (SR and normal observers), Filter Orientation (horizontal and vertical), and Filter Bandwidth (in 6 steps from $15^{\circ}$ to $90^{\circ}$ ). Mauchley's Test of Sphericity rejects the null hypothesis that all response variables have equal variance $\left(\chi^{2}(65)=120.25, p<.0001\right)$; we therefore Greenhouse-Geisser-corrected the obtained p-values( $\varepsilon$ ) to correct for the violation of this assumption.

\begin{tabular}{|c|c|c|c|c|c|c|}
\hline & $\begin{array}{l}\text { Sum of } \\
\text { Squares }\end{array}$ & DF & MeanSq & $\mathbf{F}$ & $\mathbf{p}$ & $\varepsilon$-corrected $p$ \\
\hline Group & 0.19 & 1 & 0.19 & 5.31 & 0.03 & 0.03 \\
\hline Error & 1.46 & 40 & 0.04 & & & \\
\hline Filter Orientation & 2.56 & 1 & 2.56 & 190.64 & $\mathrm{p}<.01$ & $\mathrm{p}<0.01$ \\
\hline Group X Filter Orientation & 0.04 & 1 & 0.04 & 2.85 & 0.10 & 0.10 \\
\hline Error & 0.54 & 40 & 0.01 & & & \\
\hline Filter Bandwidth & 4.80 & 5 & 0.96 & 95.81 & $\mathrm{p}<.01$ & $p<0.01$ \\
\hline Group X Filter Bandwidth & 0.08 & 5 & 0.02 & 1.66 & 0.15 & 0.17 \\
\hline Error & 2.00 & 200 & 0.01 & & & \\
\hline Filter Orientation X Filter Bandwidth & 0.68 & 5 & 0.14 & 19.76 & $\mathrm{p}<.01$ & $p<0.01$ \\
\hline $\begin{array}{l}\text { Group X Filter Orientation X Filter } \\
\text { Bandwidth }\end{array}$ & 0.01 & 5 & 0.00 & 0.19 & 0.96 & 0.95 \\
\hline Error & 1.38 & 200 & 0.01 & & & \\
\hline
\end{tabular}

